\title{
Analysis of ac magnetic susceptibility data of a room temperature superconductor
}

\author{
J. E. Hirsch \\ Department of Physics, University of California, \\ San Diego, La Jolla, CA 92093-0319, USA \\ Email: jhirsch@ucsd.edu
}

\begin{abstract}
In Ref. [1] Snider et al. reported room temperature superconductivity in carbonaceous sulfur hydride (CSH) under high pressure. Recently the data for the temperature dependent ac magnetic susceptibility shown in figures of Ref. [1] have appeared in the form of tables corresponding to different pressures [2]. Here we provide an analysis of the data for a pressure of $160 \mathrm{GPa}$. This work was performed in collaboration with D. van der Marel.
\end{abstract}

Keywords: hydride superconductor; room temperature superconductor; pressure; ac magnetic susceptibility; raw data; background signal

\section{INTRODUCTION}

In Ref. [1] it is reported that a material termed carbonaceous sulfur hydride (hereafter called CSH) is a room temperature superconductor. Data for resistance versus temperature and ac susceptibility versus temperature at six different pressures show drops suggesting superconducting transitions. Recently two of the authors of Ref. [1] have posted the numerical values of the data for the ac susceptibility curves (hereafter $\chi^{\prime}(T)$ ) published in Ref. [1] as well as the underlying raw data on arXiv [2]. The raw data and data are called "Measured Voltage" and "Superconducting Signal" respectively in Ref. [2]. Here we give an analysis of the ac susceptibility data for pressure $p=160 \mathrm{GPa}$ [3]. Other analysis of the susceptibility data in Ref. [2] were presented in Refs. [46]. The analysis presented in Sect. II of this paper was presented earlier in Ref. [7].

\section{ANALYSIS OF THE 160 GPA DATA}

Fig. 1a shows the data for $\chi^{\prime}(T)$ for one of the curves shown in Extended Data Figure 7d of Ref. [1], corresponding to pressure $160 \mathrm{GPa}$. The numerical values are given in the second column of Table 5 of Ref. [2] (labeled "Superconducting Signal"). A superconducting transition appears to take place around $T=170 \mathrm{~K}$. In Fig. 1 panels $\mathbf{c}$ and $\mathbf{d}$ these data are shown on a 15 times expanded $y$-axis. Because of the steep rise at $170 \mathrm{~K}$ the regions above and below $170 \mathrm{~K}$ need to be displayed in separate panels. A similar zoom of the $160 \mathrm{GPa}$ curve was previously shown in Fig. 9 of Ref. [6]. One of the striking features is a series of discontinuous steps. These steps are directly visible to the eye in the temperature ranges where $\chi^{\prime}(T)$ has a weak temperature dependence. However, they are also present in the range where $\chi^{\prime}(T)$ rises steeply as a function of temperature, as can be seen by calculating the difference between neighboring points

$$
\Delta \chi(j)=\chi\left(T_{j}\right)-\chi\left(T_{j-1}\right)
$$
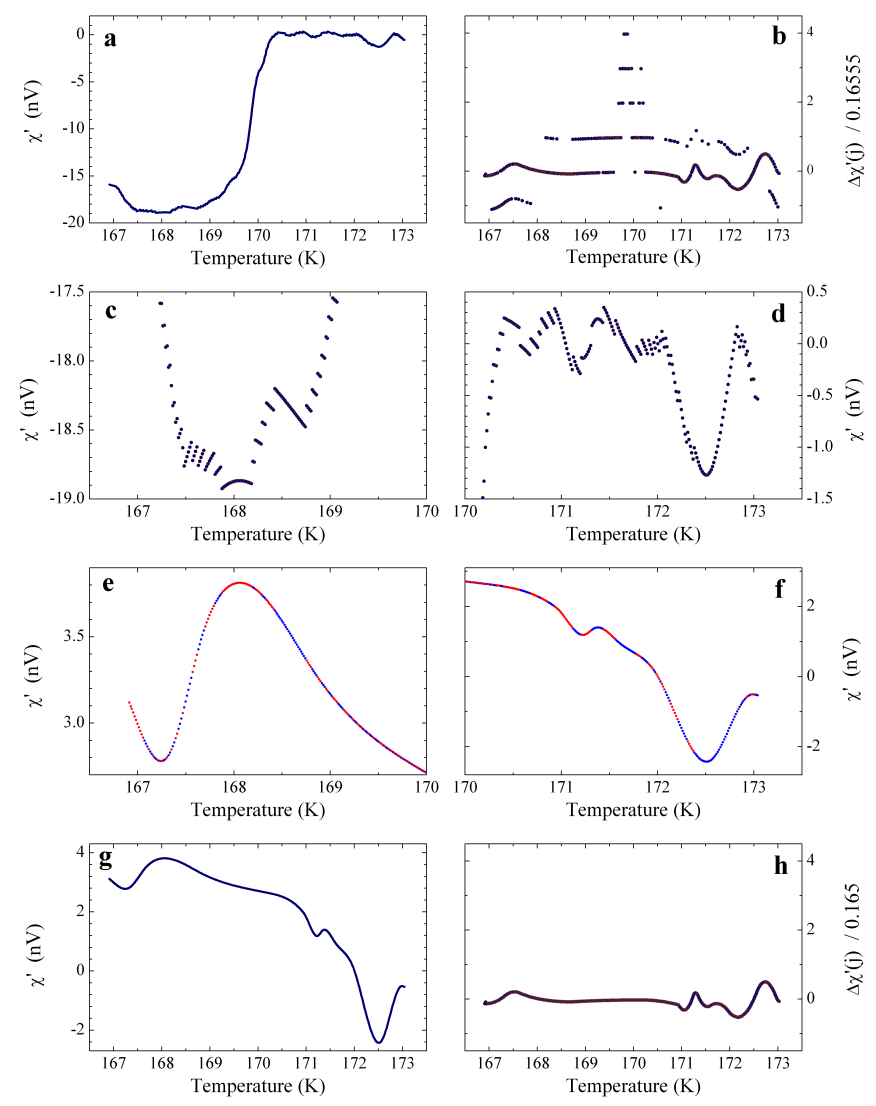

FIG. 1: a, Susceptibility data ("Superconducting Signal") for $\mathrm{CSH}$ at pressure $160 \mathrm{GPa}$, from the numerical data of Table 5 of Ref. [2]. b, The difference between neighboring points of panel a divided by 0.16555 . $\mathbf{c}$ and $\mathbf{d}$, The data of panel $\mathbf{a}$ on an enlarged scale. $\mathbf{e}, \mathbf{f}$ and $\mathbf{g}$, The data of panels $\mathbf{c}, \mathbf{d}$ and $\mathbf{a}$ after unwrapping with integer multiples of 0.16555 . The different colors of panels $\mathbf{e}$ and $\mathbf{f}$ refer to disconnected segments of panels $\mathbf{c}$ and $\mathbf{d}$. $\mathbf{h}$, Same as panel $\mathbf{b}$ but now using the unwrapped data of panel $\mathbf{g}$. The same vertical scale is used as for panel $\mathbf{b}$.

This quantity, shown in Fig. $1 \mathbf{b}$, exhibits an intriguing "aliasing" effect in the "shadow curves" displaced vertically by integer multiples of 0.16555 . To make 
this crisp, the vertical axis of Fig. $1 \mathbf{b}$ corresponds to $\Delta \chi(j) / 0.16555$. Clearly this is a set of curves vertically offset by an integer $n=-1,0,1,2,3$ and 4 .The most systematic offsets in sign and size occur between $169.6 \mathrm{~K}$ and $170.1 \mathrm{~K}$.

By shifting continuous segments of the curves by an amount $0.16555 n$, with $n$ integers that can be read off from Fig. $1 \mathbf{b}$, it is a simple and straightforward task to 'unwrap' the vertical offsets [8]. The result for the two separate ranges above and below $170 \mathrm{~K}$ is displayed in Fig. $1 \mathbf{e}$ and $\mathbf{f}$, and for the full range in panel g. Comparing panel e to $\mathbf{c}$, and $\mathbf{f}$ to $\mathbf{d}$ it is possible to verify that the resulting curves are extremely smooth and completely free of discontinuities. Comparing panel $\mathbf{g}$ to $\mathbf{a}$ the steep rise at $170 \mathrm{~K}$ is absent from panel $\mathrm{g}$. As a consistency check $\Delta \chi(j)$ was finally calculated, corresponding to panel g. Comparing the result shown in panel $\mathbf{h}$ with that in panel $\mathbf{b}$ (shown with the same vertical scale to facilitate comparison) it can be seen that there are no shadow curves in panel $\mathbf{h}$, demonstrating that not only the temperature dependence of panel $\mathbf{g}$ is smooth, the differential shown in panel $\mathbf{h}$ is, surprisingly for an experimental quantity, also completely smooth.

The behavior of the data shown in Fig. $1 \mathbf{c}$ and $\mathbf{d}$, together with the fact that the segments can be joined by vertical shifts that are all of the same form $(0.16555 \pm$ $0.00005) n$, indicates that the disconnected segments are portions of a continuous curve that has been broken up by quantized steps. The sequence of steps form together a quantized component which is entirely responsible for the steep rise of $\chi^{\prime}(T)$ at $170 \mathrm{~K}$ seen in Fig. 1 a. The data (Superconducting Signal) of Fig. 1 a can be expressed as:

$$
\begin{aligned}
\text { Superconducting Signal } & =\text { quantized component } \\
& + \text { unwrapped curve }
\end{aligned}
$$

where the unwrapped curve is given in Fig. 1 g. Fig. 2 shows the same information as panels a-d of Fig. 1 for the quantized component. The connected segments are now horizontal, and the increments in panel $\mathbf{b}$ are integers.

According to Ref. [1], a background signal measured at $108 \mathrm{GPa}$ was subtracted from the raw data (Measured Voltage) in obtaining the published data (Superconducting Signal) in Ref. [1]. In other words,

$$
\begin{aligned}
\text { Superconducting Signal } & =\text { Measured Voltage } \\
& - \text { Background Signal }
\end{aligned}
$$

Comparison of Eq. (3) and Eq. (2) strongly suggests that the Measured Voltage and background signal in Eq. (3) correspond to the quantized component and $(-1) \times$ unwrapped curve in Eq. (2) respectively.

\section{A POSSIBLE EXPLANATION OF THESE RESULTS?}

To begin to understand these results we have to understand (a) why the Measured Voltage deduced in section II
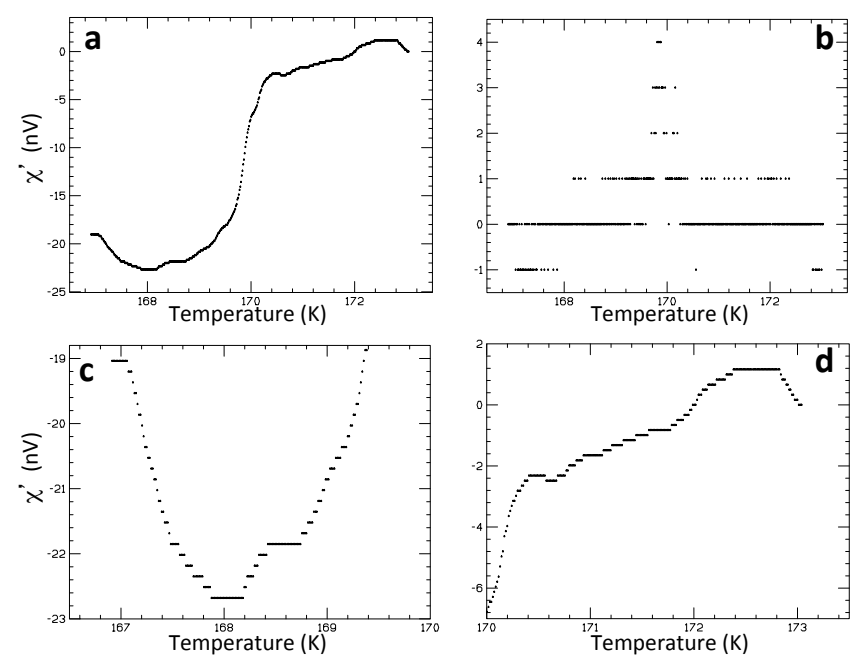

FIG. 2: a, Quantized component of susceptibility data ("Superconducting Signal") for CSH at pressure $160 \mathrm{GPa}$. b, The difference between neighboring points of panel a divided by 0.16555. $\mathbf{c}$ and $\mathbf{d}$, The data of panel a on an enlarged scale.

(quantized component) is a series of flat steps separated by jumps of a fixed magnitude $0.16555 \mathrm{nV}$, and (b) why the background signal deduced in section II (the negative of the unwrapped curve) is a smooth curve with no experimental noise.

(a) A digital lock-in amplifier will yield discrete values for the measured voltages, where the size of the step between neighboring values of measured voltages is given by the instrumental resolution. Given our conclusion in Sect. II that the quantized component shown in Fig. 2a could be the raw data (Measured Voltage), this would indicate that the resolution of the instrument in this measurement was of order $0.2 \mathrm{nV}$. Such a low resolution could result from setting the digitizer range of the lock-in amplifier to a large value, approximately $100 \mu \mathrm{V}$ [9].

(b) The smooth behavior of the background signal $((-1) \times$ panel $g$ of Fig. 1) could be explained if, rather than measured values of the background signal, a polynomial fit to the measured values was subtracted from the raw data. We note however that Ref. [1] does not mention such a procedure.

\section{RELATION WITH THE REPORTED RAW DATA}

In the previous section we have concluded that a possible way to understand the very unusual nature of the susceptibility data for $160 \mathrm{GPa}$ reported in Ref. [1] could be if the measured raw data are the quantized component of the Superconducting Signal shown in panel a of Fig. 2, and the background signal is given by the negative of the unwrapped curve panel $\mathbf{g}$ of Fig. 1. On the other hand, the superconducting signal as well as the measured raw data were reported in Ref. [2] table 5, and we can infer 

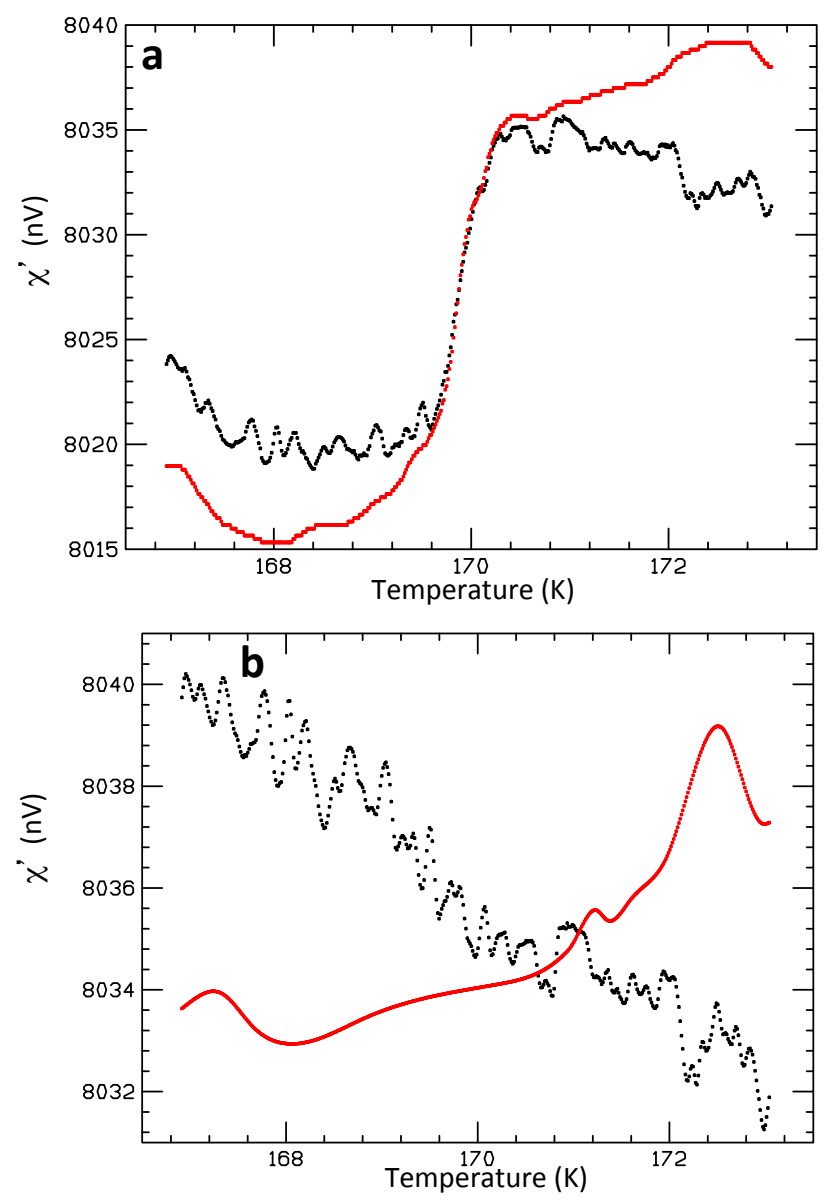

FIG. 3: a, Raw data (Measured Voltage) reported in Ref. [2] for $160 \mathrm{GPa}$ (black points), compared with quantized component of susceptibility data (red points). b, Background signal inferred by subtraction of reported raw data and data in Ref. [2] (black points), compared with background signal inferred from unwrapping of the susceptibility data (red points).

from them the background signal simply by subtraction.

Therefore, in Figs. $3 \mathbf{a}$ and $\mathbf{b}$ we compare the reported raw data and the background signal inferred from the reported raw data and the reported data [2] with our hypothesized raw data and background signal deduced in section II.

It can be seen in Fig. 3 that there is a complete disconnect between the raw data and the background signal inferred from the numbers reported in Ref. [2], and the raw data and background signal inferred from the analysis of the Superconducting Signal [1] (numerical values given in ref. [2]) discussed in section II. In particular, there is certainly no way that a polynomial fit of the black points in Fig. 3b would have any resemblance to the red curve shown in Fig. 3b, and there is a significant difference between the black and red curves in Fig. 3a. There is also no quantization of measured voltages in the raw data reported in Ref. [2]. The reported measured values of the Measured Voltage are given in Table 5 of
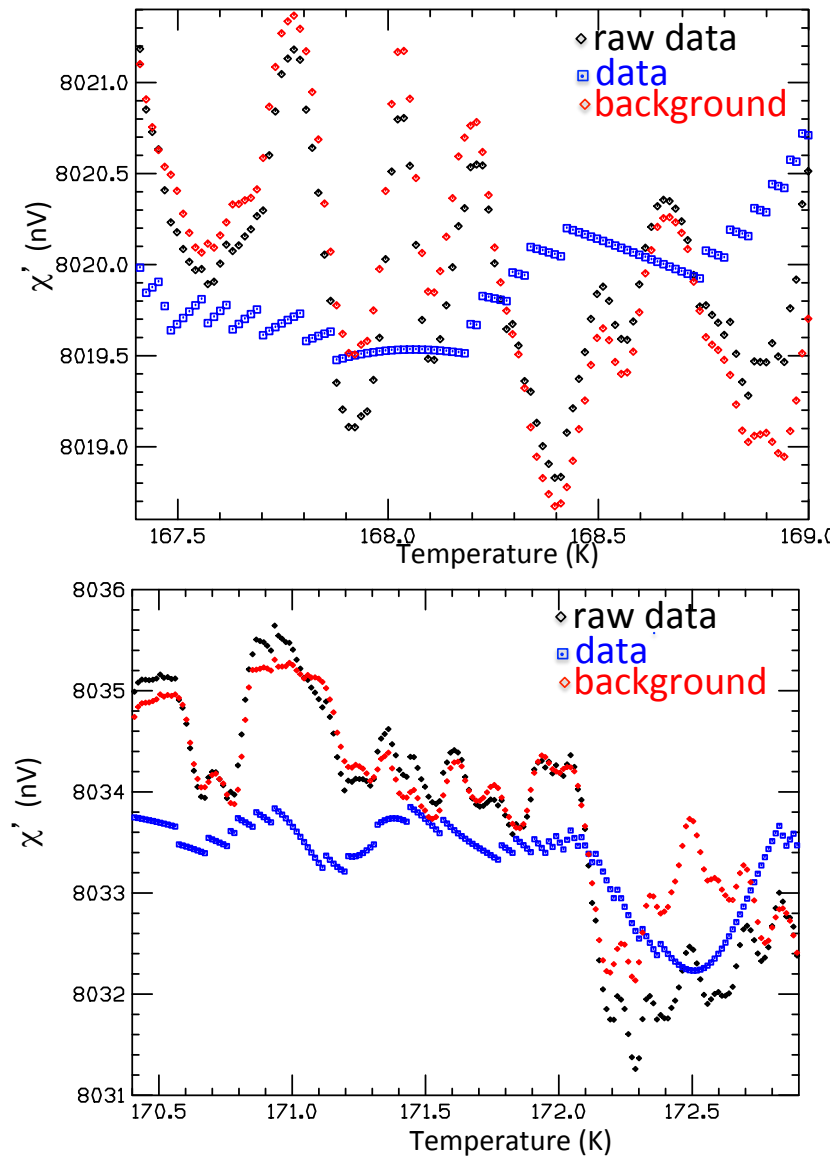

FIG. 4: Raw data, data and background signal inferred by subtraction, obtained from the numerical values reported in Table 5 of Ref. [2], for the low and high temperature regions of the $160 \mathrm{GPa}$ data shown in panels $\mathbf{c}$ and $\mathbf{d}$ of Fig. 1 .

ref. [2] with 11 significant digits, corresponding to an experimental resolution of $0.0001 \mathrm{nV}$. This is about three orders of magnitude higher resolution than the resolution of the measuring device that would yield the quantized component (red curve in Fig. 3a) as measured raw data.

It can also be seen in Fig. 3 that there is much larger noise in the raw data and background signal reported in Ref. [2] than there is in the red curves that were deduced from the reported Superconducting Signal in section II. The fact that the reported Superconducting Signal is significantly less noisy than the reported raw data was already noted in Ref. [6], not only for pressure $160 \mathrm{GPa}$ but for all other pressures as well. This is independent of the unwrapping analysis discussed in the earlier sections. In Fig. 4 we show the raw data, the data, and the background signal obtained from the values reported in table 5 of Ref. [2], for the low and high temperature parts of the $160 \mathrm{GPa}$ data. It can be seen that in order for the data (blue points) to result from subtraction of a background (red points) from raw data (black points) the oscillations in the background signal, presumably arising from instrumental noise, have to closely track oscillations 


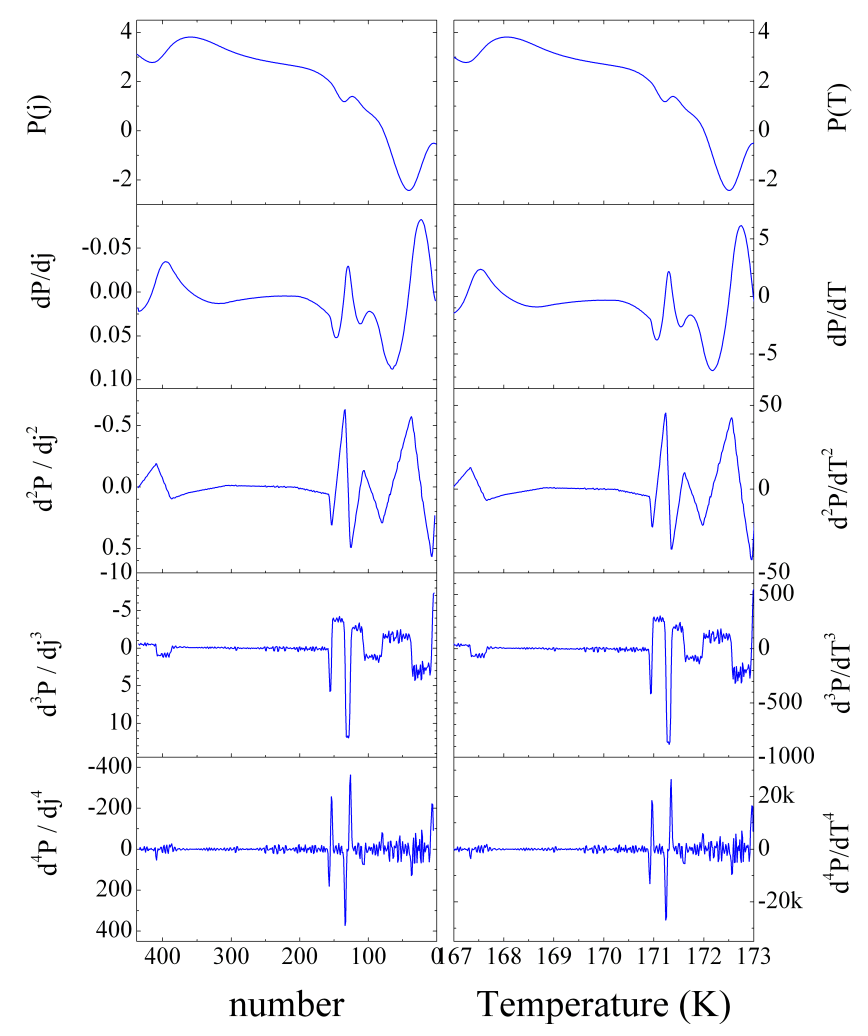

FIG. 5: From top to bottom: Unwrapped component of the susceptibility and it's first, second, third and fourth derivatives. Left panels: Numerical derivatives with respect to the row number. Right panels: Numerical derivatives with respect to the temperature.

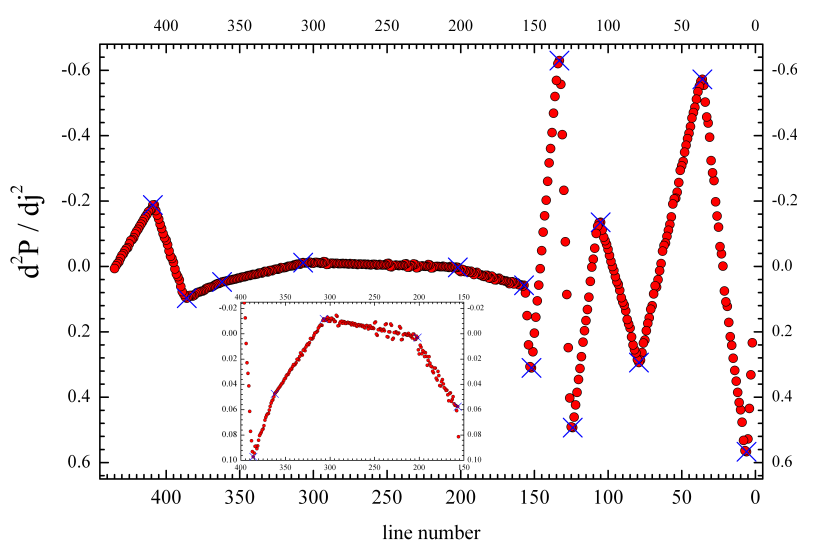

FIG. 6: Enlarged third right panel from the top of Fig. 5, showing the fourteen linear segments for the second derivative. The inset shows the center part enlarged further.

in the raw data. Independently measured raw data and background signal do not have that property.

\section{PROPERTIES OF THE UNWRAPPED CURVE}

The remarkable smoothness of the unwrapped curve obtained through the analysis in section II is illustrated by Fig. 5 where this curve is displayed together with the first, second, third and fourth derivate. The $n$ 'th derivative of the $j$ 'th point were calculated by applying the linear regression expression to the three $n-1$ 'th derivatives at $j$ and $j \pm 1$. Only at the fourth derivative is too noisy for readout. In the second derivative graph, shown in more detail in Fig. 6, all segments are straight lines. This clearly demonstrates that this curve is a chain of 14 polynomials of order 3 .

The temperatures are not equally spaced. To check whether the underlying functional dependence of the smooth curve is better described by temperature or by row number, the numerical derivatives with respect to $T$ (left), are compared to those with respect to row number $j$ (right). The similarity of the noise level for third and fourth derivative between right hand panel indicates that the 14 segments of the unwrapped curve are equally described as functions of the row number or the temperature. The coefficients using the row number representation are given in Table I.

\section{SUMMARY}

The ac susceptibility reported in Ref. [1] was obtained by measuring the "Measured Voltage", obtaining from measurements a "Background Signal", and calculating from this the reported "Superconducting Signal" = "Measured Voltage"- "Background Signal". In this paper we have analyzed in detail the published "Superconducting Signal" and "Measured Voltage" at $160 \mathrm{GPa}$.

1. We have shown that the reported "Superconducting Signal" is the sum of two components: (i) A multi segment polynomial composed of 14 third degree polynomials, and (ii) a quantized component with $y$ values binned as $y=0.16555 n(-7<n<$ 138).

2. It is not clear what causes the quantization in $0.16555 \mathrm{nV}$ steps. The variation as a function of temperature of the "Measured Voltage" reported in Ref. [2] occurs on a scale $\sim 0.001 \mathrm{nV}$ which is three orders of magnitude below $0.16555 \mathrm{nV}$.

3. We cannot identify the quantized component with the raw data since, other than containing the steep rise at $170 \mathrm{~K}$, it departs strongly from the raw data reported in Ref. [2].

4. We cannot identify the multi segment polynomial with the background signal, since it departs 


\begin{tabular}{|l|l|l|r|r|r|r|}
\hline segment & $j_{1}$ & $j_{2}$ & $\mathrm{a}$ & $\mathrm{b}$ & $\mathrm{c}$ & $\mathrm{d}$ \\
\hline 1 & 0 & 6 & -0.53416 & +0.01102 & $-1.44652 \cdot 10^{-4}$ & $-2.31481 \cdot 10^{-4}$ \\
2 & 6 & 36 & -0.6277 & +0.04915 & -0.00595 & $+8.97201 \cdot 10^{-5}$ \\
3 & 36 & 79 & 6.0351 & -0.50047 & +0.00911 & $-4.70588 \cdot 10^{-5}$ \\
4 & 79 & 105 & -34.79958 & +1.05692 & -0.01069 & $+3.68086 \cdot 10^{-5}$ \\
5 & 105 & 124 & 111.80933 & -3.07543 & +0.02813 & $-8.47911 \cdot 10^{-5}$ \\
6 & 124 & 133 & -760.25698 & +17.83366 & -0.13897 & $+3.60385 \cdot 10^{-4}$ \\
7 & 133 & 152 & 374.72152 & -7.77894 & +0.05369 & $-1.22696 \cdot 10^{-4}$ \\
8 & 152 & 157 & -811.29603 & +15.52318 & -0.09892 & $+2.10487 \cdot 10^{-4}$ \\
9 & 157 & 202 & -21.72553 & +0.33995 & -0.0016 & $+2.5448 \cdot 10^{-6}$ \\
10 & 202 & 307 & -1.83508 & +0.05288 & $-2.20374 \cdot 10^{-4}$ & $+3.35069 \cdot 10^{-7}$ \\
11 & 307 & 362 & 77.69287 & -0.73011 & +0.00235 & $-2.47422 \cdot 10^{-6}$ \\
12 & 362 & 386 & 201.88584 & -1.75985 & +0.0052 & $-5.09699 \cdot 10^{-6}$ \\
13 & 386 & 409 & -2032.86288 & +15.55903 & -0.03954 & $+3.34264 \cdot 10^{-5}$ \\
14 & 409 & 437 & 1596.02048 & -11.07287 & +0.02561 & $-1.97026 \cdot 10^{-5}$ \\
\hline
\end{tabular}

TABLE I: Coefficients of the expression $\chi=a+b j+c j^{2}+d j^{3}$ where $j$ is the row number for the 14 segments defined by $j_{1} \leq j \leq j_{2}$. The coefficients were obtained by least square fitting the corresponding segments to a third polynomial. The units are $n V$.

strongly from the difference "Measured Voltage""Superconducting Signal" according to the data reported in Ref. [2].

5. All in all it is not clear how the ac susceptibility data reported in Ref. [1] were obtained from the measured raw data reported in Ref. [2].

For readers that would like to check the analysis resulting in Fig. 1 and Fig. 5 of this paper we made the corresponding excel tables available in Ref. [8]. Numerical data for all pressures reported in Ref. [2] in image format are given in text format in Ref. [10].

\section{Acknowledgments}

This work was performed in collaboration with D. van der Marel, who chose not to be listed as an author for personal reasons. We are grateful to Brad Ramshaw and Peter Armitage for stimulating discussions.

Note added: After completion of this paper, we became aware of the recently posted arXiv:2201.11883 [11]. It will become possible to address the issues raised in that paper after the authors release the numerical values for their UDB_1 [11] ("user defined background method 1 ") along with a detailed documentation of how this is derived -and can be reproduced by others- from the experimental data.
[1] E. Snider, N. Dasenbrock-Gammon, R. McBride, M. Debessai, H. Vindana, K. Vencatasamy, K. V. Lawler, A. Salamat and R. P. Dias, "Room-temperature superconductivity in a carbonaceous sulfur hydride", Nature 586, 373 (2020).

[2] R. P. Dias and A. Salamat, "Standard Superconductivity in Carbonaceous Sulfur Hydride", arXiv:2111.15017, Dec. 28, 2021.

[3] The analysis presented in this paper was performed in its entirety in collaboration with D. van der Marel.

[4] J. E. Hirsch, "Disconnect between published ac magnetic susceptibility of a room temperature superconductor and measured raw data", doi: 10.20944/preprints202112.0115.v2.

[5] J. E. Hirsch, "Incompatibility of published ac magnetic susceptibility of a room temperature superconductor with measured raw data", doi: 10.20944/preprints202112.0188.v2.
[6] J. E. Hirsch, "Superconductivity in Carbonaceous Sulfur Hydride: Further Analysis of Relation between Published AC Magnetic Susceptibility Data and Measured Raw Data", doi: 10.20944/preprints202201.0003.v1.

[7] D. van der Marel and J. E. Hirsch, "Comment on Nature 586, 373 (2020) by E. Snider et al", arXiv:2201.07686 (2022).

[8] Excel files with the quantities shown in Fig. 1 and Fig. 5 are publicly available on http://dirkvandermarel.ch/wp-content/uploads/chi.xlsx and http://dirkvandermarel.ch/wpcontent/uploads/derivchi.xlsx.

[9] Brad Ramshaw, private communication.

[10] Numerical values for all the data given in the tables of Ref. [2] are publicly available on https://jorge.physics.ucsd.edu/cshdata.html.

[11] R. P. Dias and A. Salamat, "Reply to "Comment on Nature 586, 373 (2020) by E. Snider et al."”, 
arXiv:2201.11883 (2022). 